\title{
RADIO FREQUENCY ELECTROMAGNETIC RADIATION (RF-EMR) FROM GSM (0.9/1.8GHZ) MOBILE PHONES INDUCES OXIDATIVE STRESS AND REDUCES SPERM MOTILITY IN RATS
}

\author{
Maneesh Mailankot, ${ }^{\text {I Anil P Kunnath, }}$, Jayalekshmi H,, ${ }^{\text {III }}$ Bhargav Koduru, \\ Rohith Valsalan $^{\mathrm{v}}$
}

doi: 10.1590/S1807-59322009000600011

\begin{abstract}
Mailankot M, Kunnath AP, I Jayalekshmi H, Koduru B, Valsalan R. Radio frequency electromagnetic radiation (RF-EMR) from gsm (0.9/1.8ghz) mobile phones induces oxidative stress and reduces sperm motility in rats. Clinics. 2009;64(6):561-5.
\end{abstract}

INTRODUCTION: Mobile phones have become indispensable in the daily lives of men and women around the globe. As cell phone use has become more widespread, concerns have mounted regarding the potentially harmful effects of RF-EMR from these devices.

OBJECTIVE: The present study was designed to evaluate the effects of RF-EMR from mobile phones on free radical metabolism and sperm quality.

MATERIALS AND METHODS: Male albino Wistar rats (10-12 weeks old) were exposed to RF-EMR from an active GSM (0.9/1.8 $\mathrm{GHz}$ ) mobile phone for 1 hour continuously per day for 28 days. Controls were exposed to a mobile phone without a battery for the same period. The phone was kept in a cage with a wooden bottom in order to address concerns that the effects of exposure to the phone could be due to heat emitted by the phone rather than to RF-EMR alone. Animals were sacrificed 24 hours after the last exposure and tissues of interest were harvested.

RESULTS: One hour of exposure to the phone did not significantly change facial temperature in either group of rats. No significant difference was observed in total sperm count between controls and RF-EMR exposed groups. However, rats exposed to RF-EMR exhibited a significantly reduced percentage of motile sperm. Moreover, RF-EMR exposure resulted in a significant increase in lipid peroxidation and low GSH content in the testis and epididymis.

CONCLUSION: Given the results of the present study, we speculate that RF-EMR from mobile phones negatively affects semen quality and may impair male fertility.

KEYWORDS: Mobile phone; Electromagnetic radiation; Sperm motility; Oxidative Stress; Rats.

\section{INTRODUCTION}

Concerns about the possible health effects of mobile phone usage are growing as the number of users has increased tremendously over the past several years. Mobile

\footnotetext{
${ }^{\text {I }}$ Department of Biochemistry, Melaka Manipal, Medical College (Manipal Campus), Manipal, India.

II College of Allied Health Sciences, Kasturba Medical College, Manipal, India.

III Amritha School of Biotechnology, Clappana, Kerala, India.

${ }^{\text {IV }}$ Department of Biochemistry, Kasturba Medical College, Manipal, India.

${ }^{v}$ Department of Medicine, Kasturba Medical College, Manipal, India.

Email: manu_only@hotmail.com

Tel: 918202922637

Received for publication on January 05, 2009

Accepted for publication on March 06, 2009
}

phone technology uses radiofrequency electromagnetic radiation (RF-EMR) and has drastically increased the RF-EMR exposure encountered in daily life. Many recent studies have raised questions regarding the safety of such RF-EMR exposure. For example, microwaves generated by mobile phones have been linked to several genetic defects. ${ }^{1-5}$ Research also suggests that microwave radiation from mobile phones may induce chromosomal instability and lead to increased cancer risk. ${ }^{3,6,7}$ Our previous studies have shown that RF-EMR from a $0.9 / 1.8 \mathrm{GHz}$ mobile phone is anxiogenic (Kumar et al., in press) and impairs avoidance behavior (unpublished data) and spatial memory performance (Sareesh et al., in press) in rats.

As men usually carry mobile phones in their pockets or 
in holders close to their reproductive organs, it is important to evaluate the effects of mobile phone use on male fertility. Although many recent epidemiological studies ${ }^{8-12}$ have suggested that mobile phone use may play a role in male infertility, the mode of action on the male reproductive system remains unclear. Mobile phones might impact the reproductive system via a RF-EMR-specific effect, a thermal effect, or a combination of these effects. In contrast it has recently been suggested that the antioxidant effect of alpha lipoic acid may improve sperm quality. ${ }^{13}$ Therefore, carefully designed studies are needed to properly assess the effects of cell phone use on male fertility.

In the present study, we showed that RF-EMR from a mobile phone in active mode disturbs free radical metabolism in reproductive tissue in vivo and thereby leads to changes in semen parameters. Our results suggest a causal relationship between RF-EMR and changes in semen quality.

\section{MATERIALS AND METHODS}

Animals: Albino male Wistar rats aged 10-12 weeks were housed in plastic cages (14" $\times 9$ " $\times 8$ ", 3 rats per cage) inside a well-ventilated room kept at $22 \pm 2^{\circ} \mathrm{C}$ with a 12 -hr light:dark cycle. All animals had free access to a standard diet and water. Animals were divided into a control group (group I) and an RF-EMR-exposed group (group II) with 6 rats per group. The Animal Ethics Committee (Manipal University, Manipal) approved all procedures.

Mobile phone exposure: Group II animals were exposed to RF-EMR from a GSM $(0.9 / 1.8 \mathrm{GHz})$ mobile phone in active mode for 1 hour continuously per day for 28 days. To address the concern that any effects of the mobile phone could be due to heat emitted rather than to RF-EMR alone, the phone was kept in a 4 " $\times 2$ " $\times 1$ " cage with a wooden bottom. Animals were free to move about in the cage during the exposure period and food and water were available $a d$ libitum. Control animals were exposed to a mobile phone without battery in a similarly sized cage for the same period in a separate, similar room. Both cages were brought back to the home room following phone exposure.

After the experimental period, all animals were killed by cervical dislocation. The tissues of interest were harvested via incisions in the scrotum and subsequent dissection of the testis and epididymis.

Determination of motile sperm count: The caudal epididymis was excised, placed in a sperm collection vial containing $5 \mathrm{~mL}$ BSA-Hanks solution, and cut three times to release the sperm. A $0.05-\mathrm{mL}$ sample of the sperm fluid was removed and diluted with $0.95 \mathrm{~mL}$ of BSA-Hanks solution. The number of non-motile sperm in the diluted sperm fluid was determined with a hemocytometer. After the non-motile sperm had been counted, the vessel containing the diluted sperm fluid was immersed in hot water to kill the motile sperm. The fluid was returned to room temperature and the total number of sperm was counted with the hemocytometer. When gross observation showed a small sperm population in the sperm fluid (low turbidity), an undiluted aliquot of the fluid was used. The percentage of motile sperm was calculated using the following equation:

Motile sperm rate $(\%)=\frac{\begin{array}{c}\text { (number of sperm }- \text { number of } \\ \text { non-motile sperm) }\end{array}}{\text { Number of sperm }} \times 100$

Determination of total sperm count: The caudal epididymis used in the above procedure was further cut to release all sperm, and the fluid in the vial was filtered through a nylon-mesh sieve. The resulting stock filtrate was then diluted with formalinized saline $(0.1 \mathrm{ml}$ filtrate in $1.9 \mathrm{ml}$ saline), and the number of sperm was determined with a hemocytometer. When the sperm population in the sample was too small (low turbidity), the stock filtrate was not diluted; instead, the vessel was directly immersed in hot water and returned to room temperature before the number of sperm was determined. The measured number of sperm was multiplied by the dilution factor to yield the total sperm count.

Sperm count $=$ Measured number of sperm $\times$ Dilution factor

Determination of reduced glutathione (GSH): GSH concentration was determined as described by Cui and $\mathrm{Lou}^{14}$. Briefly, $10 \mu \mathrm{L}$ of supernatant from tissue homogenized in $10 \%$ TCA (trichloroacetic acid) was mixed with $10 \mu \mathrm{L}$ of 5,5'-dithio-bis(2-nitrobenzoic acid) (DTNB) in $2.0 \mathrm{mg} / 2.5$ $\mathrm{mL}$ methanol. The volume was adjusted to $200 \mu \mathrm{L}$ with 1.0 M Tris-HCl, pH 8.2 containing 0.02 M EDTA. GSH concentration was quantified by measuring the absorbance of the reaction product at $412 \mathrm{~nm}$ (compared to a standard curve of GSH).

Determination of lipid peroxidation (LPO): Oxidative stress induction was indicated by the amount of LPO. LPO was quantified by measuring the formation of thiobarbituric acid reactive substances, ${ }^{15}$ expressed as malondialdehyde equivalents (nmol MDA/mg tissue), with 1,1 3,3-tetramethoxypropane used as a standard. After weighing, tissues were homogenized in ice-cold 0.25 M Tris $\mathrm{pH} 7.4$ and centrifuged. The resulting supernatant was mixed with TCA-TBA-HCl [Trichloroacetic acid (TCA): $15 \% \mathrm{w} / \mathrm{v}$, thiobarbituric acid (TBA) $0.375 \%$, hydrochloric acid $(\mathrm{HCl})$ $0.25 \mathrm{~N}$ ] and heated for $15 \mathrm{~min}$ in a boiling water bath. The lysate was cooled and centrifuged to remove the flocculent precipitate. The absorbance of the supernatant was read at $532 \mathrm{~nm}$. 
Student's t tests were performed to determine whether differences between the two groups were statistically significant. The significance threshold was set to $\mathrm{p}<0.05$.

\section{RESULTS}

Temperature: Facial temperatures did not deviate by more than $0.1^{\circ} \mathrm{C}$ after mobile phone exposure in either group. Initial facial temperatures were $32.7 \pm 0.69^{\circ} \mathrm{C}$ in the control group and $32.68 \pm 0.53^{\circ} \mathrm{C}$ in the experimental group. After $1 \mathrm{hr}$ of phone exposure, the average temperatures were $32.9 \pm 0.82^{\circ} \mathrm{C}$ in the control group and $32.83 \pm 0.58^{\circ} \mathrm{C}$ in the experimental group. Temperature differences between the groups were not statistically significant.

\section{A}

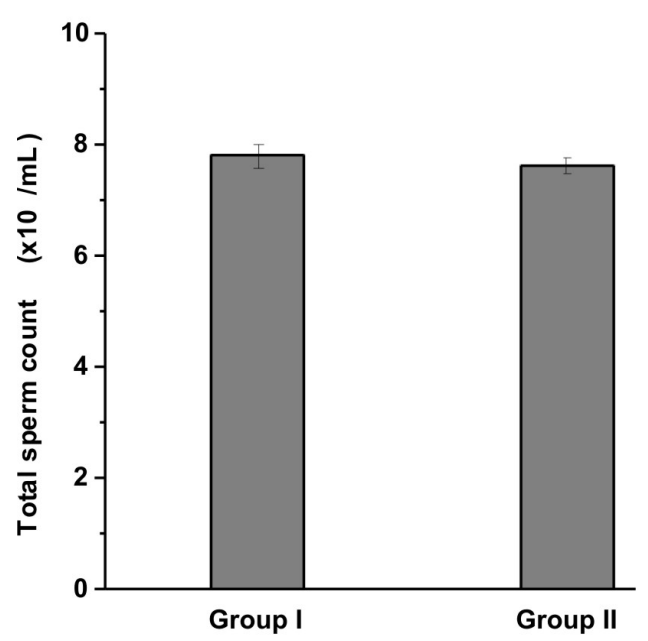

B

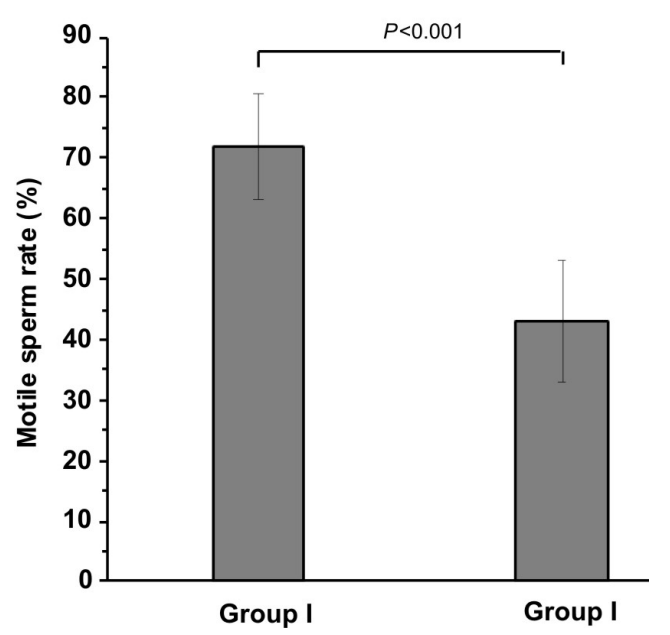

Figure 1 - Sperm count. A. Total sperm count. No significant difference was observed in the total sperm counts for the control (group I) and RFEMR exposed rats (group II). B. Percent of motile sperm. RF-EMR from a mobile phone significantly reduced the percentage of motile sperm in group II. The results are expressed as the mean \pm standard deviation of six epididymides from six rats.
Sperm parameters: The total sperm count in controls and RF-EMR-exposed animals were $7.8 \pm 0.21 \times 10^{7} / \mathrm{mL}$ and $7.6 \pm 0.13 \times 10^{7} / \mathrm{mL}$, respectively (Fig. 1A). This difference $(2.56 \%)$ was not statistically significant $(p=0.052)$.

The percent of motile sperm was significantly reduced in RF-EMR exposed animals compared with controls (Fig. 1B). The average percent of motile sperm for controls and RF-EMR exposed animals were $71.97 \pm 8.7 \%$ and $43.08 \pm$ $10.03 \%$, respectively $(p<0.002)$, a reduction of $\sim 40 \%$.

GSH content: A significant depletion of intracellular GSH was observed in the RF-EMR-exposed group, compared to the control group (Fig. 2A). The decrease was $\sim 10 \%$ in the testis and $\sim 24 \%$ in the epididymis.

Oxidative damage (LPO): Exposure to RF-EMR from
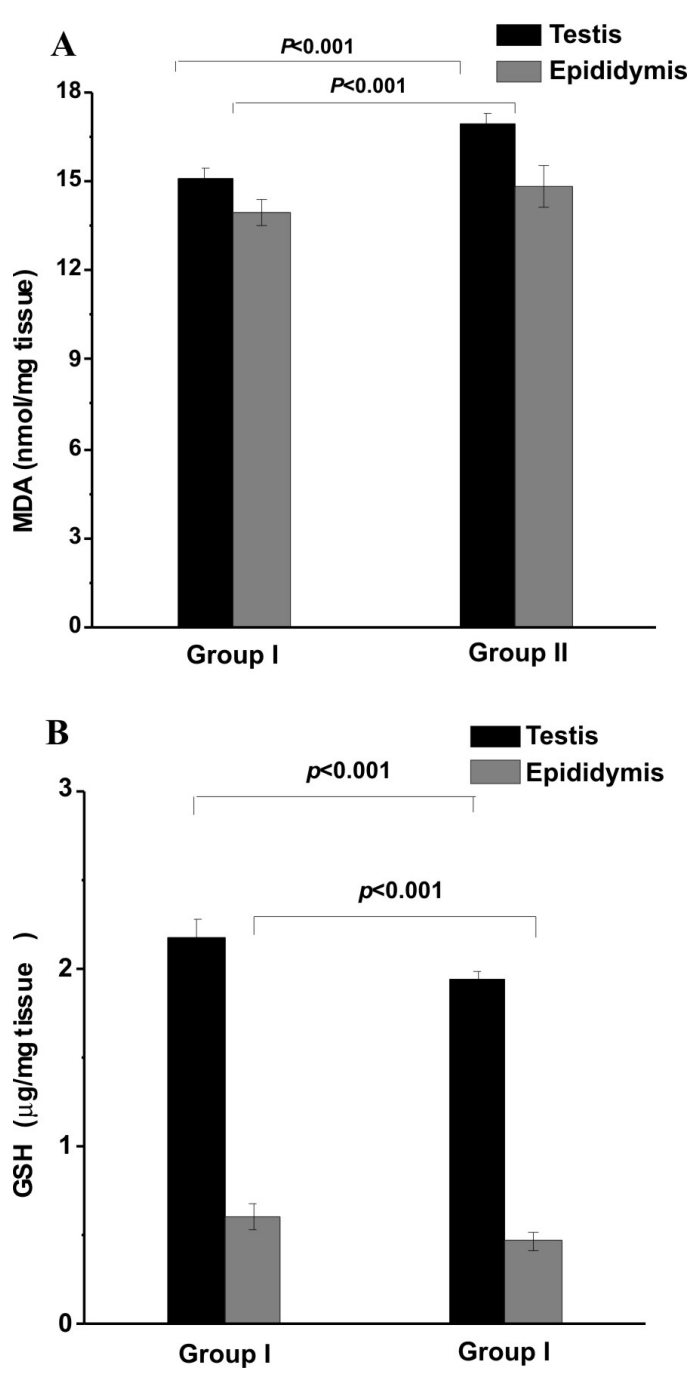

Figure 2 - LPO and GSH. A. Concentration of GSH in reproductive tissues. A significant reduction in testicular and epididymal GSH was observed in rats exposed to RF-EMR (group II) compared to controls (group I) B. Endogenous malondialdehyde (MDA) data. Significant increases were observed in both testicular and epididymal MDA in the rats exposed to RF-EMR (group II) compared to controls (group I). Results are expressed as mean \pm standard deviation of tissue isolated individually from six rats. 
the mobile phone increased endogenous MDA levels (an index of in vivo $\mathrm{LPO}$ ) in both the testis and the epididymis (Fig. 2B). The increase was $\sim 8 \%$ in testis and $\sim 12 \%$ in epididymis.

\section{DISCUSSION}

In the present study, we have tested the hypothesis that RF-EMR from mobile phones results in oxidative stress and decreases semen quality. We found a striking reduction in the percentage of motile sperm in rats exposed to RF-EMR, as well as a significantly elevated LPO and decreased GSH content in the testis and epididymis.

Several recent epidemiological studies have investigated the effects of RF-EMR from mobile phones on the human body, but results to date have been contradictory and inconclusive. Furthermore, these studies have not addressed questions regarding the thermal effects of mobile phone exposure. ${ }^{16-18}$ Many researchers believe that the effects of mobile phone exposure may be due to the cumulative effects of the heat generated and the RF-EMR emitted from mobile phone; others have suggested that the contribution of the non-thermal component is minimal and that the effects of mobile phone exposure would be negligible if the thermal effect could be eliminated. ${ }^{19-21}$ Certainly, it is widely accepted that temperature increases adversely affect sperm maturity and motility. ${ }^{16-18,22,23}$ In the present study, we excluded temperature effects by keeping the mobile phone in a smaller cage within the home cage. Furthermore, animals were allowed to move freely within the home cage in order to reduce their contact with the phone, and both cages were kept in well-ventilated rooms throughout the experiment to further reduce the possibility of heating. Finally, to confirm the lack of thermal effect, we measured the facial temperatures of rats in both groups both before and after exposure to the phone. After 1 hour of exposure, the mean facial temperature did not differ significantly from the initial temperature in either group. This experimental design eliminated mechanical heat influence from the phone, and allowed us to conclude that RF-EMR alone affected the reproductive tissue.

Oxidative stress is also a well-established cause of male infertility. ${ }^{24-26}$ Reactive oxygen species (ROS) from spermatozoa and infiltrating leukocytes cause infertility principally by affecting sperm motility. ${ }^{27,}{ }^{28}$ Spermatozoa possess a multiple plasma membrane redox system that is similar to the transmembrane NADH oxidase. NADH oxidase activity is a major source of superoxide anions ${ }^{29}$ and, interestingly, RF-EMR has been shown to stimulate NADH oxidase in the plasma membrane of mammalian cells. Normally, ROS is kept at physiologically low levels by intracellular free radical scavengers. GSH, a major thiol in living organisms, is one such scavenger, and plays a central role in coordinating the body's antioxidant defense mechanisms against free radicals. Conditions that perturb intracellular glutathione levels result in significantly altered cellular metabolism. Tissue GSH reflects tissues ability to detoxify, preserve the proper cellular redox balance, and protect cells..$^{30} \mathrm{GSH}$ also probably plays a role in sperm nucleus condensation and spindle microtubule formation. ${ }^{30}$ The depletion of GSH in RF-EMR exposed animals observed here supports our hypothesis that elevated ROS is responsible for low percentages of motile sperm. Similar findings were reported for human ejaculate exposed to RFEMR from a mobile phone. ${ }^{31} \mathrm{We}$ also believe that RF-EMR induces oxidative stress that affects testicular function and structure in rabbits exposed to mobile phones. ${ }^{32}$

Our study validates recent observational studies showing that mobile phone use may play a role in male infertility. Further research will be required to understand the long-term effects of mobile phone use.

\section{ACKNOWLEDGEMENTS}

We would like to thank Dr. Guruprasad Kalthur from the Clinical Embryology Division of Reproductive Medicine, Kasturba Medical College, Manipal, for his technical assistance.

\section{REFERENCES}

1. Aitken R, Bennetts L, Sawyer D, Wiklendt A, King B. Impact of radiofrequency electromagnetic radiation on DNA integrity in the male germline. Int J Androl. 2005;28:171-9.

2. Tice RR, Hook GG, Donner M, McRee DI, Guy AW. Genotoxicity of radiofrequency signals. I. Investigation of DNA damage and micronuclei induction in cultured human blood cells. Bioelectromagnetics. 2002;23:113-26.
3. Pacini S, Ruggiero M, Sardi I, Aterini S, Gulisano F, Gulisano M. Exposure to global system for mobile communication (GSM) cellular phone radiofrequency alters gene expression, proliferation, and morphology of human skin fibroblasts. Oncol Res. 2002;13:19-24.

4. Harvey C, French PW. Effects on protein kinase $\mathrm{C}$ and gene expression in a human mast cell line, HMC-1, following microwave exposure. Cell Biol Int. 2000;23:739-48. 
5. Goswami PC, Albee LD, Parsian AJ, Baty JD, Moros EG, Pickard WF, et al. Proto-oncogene mRNA levels and activities of multiple transcription factors in C3H 10T 1/2 murine embryonic fibroblasts exposed to 835.62 and $847.74 \mathrm{MHz}$ cellular phone communication frequency radiation. Radiat Res. 1999;151(3):300-9.

6. Mashevich M, Folkman D, Kesar A, Barbul A, Korenstein R, Jerby E, et al. Exposure of human peripheral blood lymphocytes to electromagnetic fields associated with cellular phones leads to chromosomal instability. Bioelectromagnetics. 2003;24:82-90.

7. Sykes PJ, McCallum BD, Bangay MJ, Hooker AM, Morley AA. Effect of exposure to $900 \mathrm{MHz}$ radiofrequency radiation on intrachromosomal recombination in pKZ1 mice. Radiat Res. 2001;156:495-502.

8. Agarwal A, Deepinder F, Sharma RK, Ranga G, Li J. Effect of cell phone usage on semen analysis in men attending infertility clinic: an observational study. Fertil Steril. 2008;89:124-8.

9. Davoudi M, Brossner C, Kuber W. The influence of electromagnetic waves on sperm motility. Urol Urogynecol. 2002;19:18-32.

10. Fejes I, Zavaczki Z, Szollosi J, Koloszar S, Daru J, Kovacs L, et al. Is there a relationship between cell phone use and semen quality? Arch Androl. 2005;51:385-93.

11. Wdowiak A, Wdowiak L, Wiktor H. Evaluation of the effect of using mobile phones on male fertility. Ann Agric Environ Med. 2007;14:16972.

12. Baste V, Riise T, Moen BE. Radiofrequency electromagnetic fields; male infertility and sex ratio of offspring. Eur J Epidemiol. 2008;23:36977.

13. Ibrahim Siti Fatimah, Osman Khairul, Das Srijit, Othman Abas Mazni, Majid Norzaiti Abdul, Rahman Mohd Padzil Abdul. A study of the antioxidant effect of alpha lipoic acids on sperm quality. Clinics. $2008 ; 63: 545-50$

14. Cui XL, Lou MF. The effect and recovery of long-term $\mathrm{H} 2 \mathrm{O} 2$ exposure on lens morphology and biochemistry. Exp Eye Res. 1993;57:157-67.

15. Sinnhuber RO, Yu TC, Yu TC. Characterization of the red pigment formed in the thiobarbituric acid determination of oxidative capacity. Food Res. 1958;23:626-70.

16. Sheiner EK, Sheiner E, Hammel RD, Potashnik G, Carel R. Effect of occupational exposures on male fertility: literature review. Ind Health. 2003;41:55-62.

17. Kumar S. Occupational exposure associated with reproductive dysfunction. J Occup Health. 2004;46:1-19.

18. Fejes Z, Zavaczki J, Szollosi S, Koloszar J, Daru, Kovacs L, et al. Is there a relationship between cell phone use and semen quality? Arch Androl 2005;51:385-93.

19. Black DR, Heynick LN. Radiofrequency (RF) effects on blood cells, cardiac, endocrine, and immunological functions. Bioelectromagnetics 2003;24:S187-95.
20. Jauchem JR. A literature review of medical side effects from radiofrequency energy in the human environment: involving cancer, tumors, and problems of the central nervous system. J Microw Power Electromagn Energy. 2003;38:103-23.

21. Meltz ML. Radiofrequency exposure and mammalian cell toxicity, genotoxicity, and transformation. Bioelectromagnetics 2003;24:S196213.

22. Bonde JP, Giwercman A. Ernst E. Identifying environmental risk to male reproductive function by occupational sperm studies: logistics and design options. Occup Environ Med. 1996;53:511-9.

23. Brusick D, Albertini R, McRee D, Peterson D, Williams G, Hanawalt P et al. Genotoxicity of radiofrequency radiation. Environ Mol Mutagen. 1998;32:1-16

24. Maneesh M, Dutta S, Chakrabarti A, Vasudevan DM. Alcohol abuseduration dependent decrease in plasma testosterone and antioxidants in males. Indian J Physiol Pharmacol. 2006;50:291-6.

25. Maneesh M, Jayalekshmi H, Dutta S, Chakrabarti A, Vasudevan DM. Effect of chronic ethanol administration on testicular antioxidant system and steroidogenic enzyme activity in rats. Indian J Exp Biol.2005;43:445-9.

26. Maneesh M, Jayalekshmi H, Dutta S, Chakrabarti A, Vasudevan DM. Experimental therapeutic intervention with ascorbic acid in ethanol induced testicular injuries in rats. Indian J Exp Biol. 2005;43:172-6.

27. Guthrie HD, Welch GR, Long JA. Mitochondrial function and reactive oxygen species action in relation to boar motility. Theriogenology. 2008;70:1209-15

28. Sharma R, Pasqualotto F F, Nelson D R, Thomas A J Jr, Agarwal A. Relationship between seminal white blood cell counts and oxidative stress in men treated at an infertility clinic. J Androl. 2001;22:575-83.

29. Martin-Romero FJ, Gutierrez-Martín Y, Henao F, Gutierrez-Merino C The NADH oxidase activity of the plasma membrane of synaptosomes is a major source of superoxide anion and is inhibited by peroxynitrite. J Neurochem. 2002;82:604-14.

30. Irvine DS. Glutathione as a treatment for male infertility. Reprod. 1996;1:6-12.

31. Agarwal A, Desai NR, Makker K, Varghese A, Mouradi R, Sabanegh E, et al. Effects of radiofrequency electromagnetic waves (RF-EMW) from cellular phones on human ejaculated semen: an in vitro pilot study. Fertil Steril. 2008 Sep 18. [Epub ahead of print].

32. Salama N, Kishimoto T, Kanayama HO. Effects of exposure to a mobile phone on testicular function and structure in adult rabbit. Int J Androl. 2008 Dec 2. [Epub ahead of print]. 
